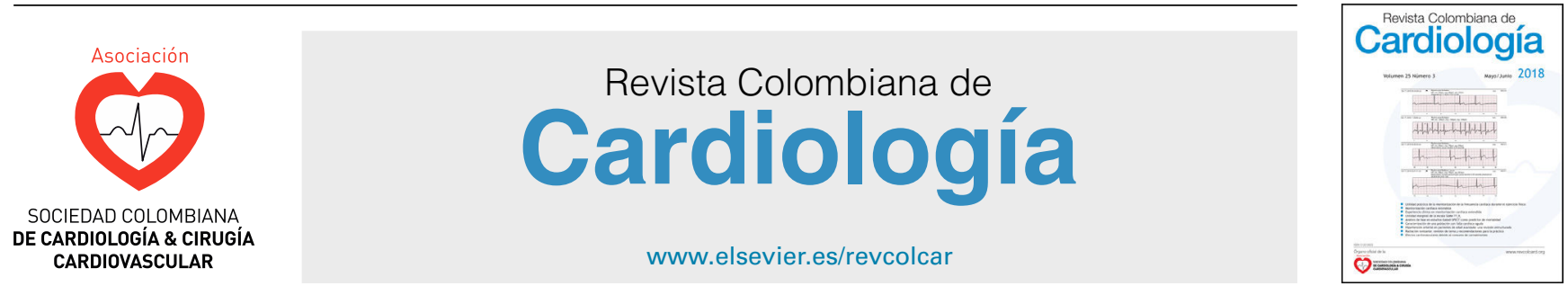

CARDIOLOGÍA DEL ADULTO - REVISIÓN DE TEMAS

\title{
Revisión de la evidencia de estudios de supervivencia de pacientes con prótesis valvular biológica
}

\section{Oneida Castañeda-Porras}

Segura, Morón \& Castañeda Asesores en Salud Ltda. (SMC-AS) Unidad de Investigaciones, Bogotá, Colombia

Recibido el 20 de abril de 2017; aceptado el 13 de mayo de 2019

Disponible en Internet el 2 de noviembre de 2019

\section{PALABRAS CLAVE \\ Prótesis valvulares \\ cardíacas; \\ Bioprótesis}

\begin{abstract}
Resumen
Introducción: las modificaciones en las características estructurales/funcionales de las prótesis valvulares han derivado en un aumento en las curvas de sobrevida/supervivencia de los pacientes, dada la reducción de las tasas de morbilidad y mortalidad posteriores a intervenciones quirúrgicas de las válvulas cardíacas y los cambios sustanciales en la relación costo-efectividad y costo-beneficio de estos procedimientos.

Objetivo: conocer la supervivencia de pacientes mayores de 60 años de edad, con prótesis valvular biológica.

Materiales y métodos: revisión de estudios de seguimiento publicados en los cuales se evaluó la supervivencia de los pacientes sometidos a reemplazo valvular. Se hizo una búsqueda de artículos científicos registrados en la base de datos PubMed, de acuerdo con los descriptores seleccionados como palabras clave - Prótesis Valvulares Cardíacas/Heart Valve Prosthesis, Bioprótesis/Bioprosthesis, Supervivencia/Survival - en el programa de gestión de referencias EndNote. Se valoró la fuerza de evidencia científica de cada uno de los artículos seleccionados con la escala de McMaster para estudios cuantitativos.

Resultados: se obtuvieron 12 referencias y se descartaron 5 (41,7\%); calificación global de "fuerte" con la escala de McMaster 7 (58,3\%). El tiempo promedio de seguimiento fue de 13,7 años y la razón de masculinidad de 1:0,8. La edad promedio de los pacientes fue 67,2 (rango $60,0-78,5)$ años. La supervivencia promedio a diez años fue del $65 \%$.

Conclusiones: se observa mayor supervivencia entre los pacientes sometidos a reemplazo valvular mecánico versus biológico; sin embargo, la literatura plantea como opción razonable el uso de prótesis biológicas en pacientes mayores de 60 años. Se reconoce, así mismo, la necesidad de hacer más estudios que puedan corroborar los hallazgos.

( 2019 Sociedad Colombiana de Cardiología y Cirugía Cardiovascular. Publicado por Elsevier España, S.L.U. Este es un artículo Open Access bajo la licencia CC BY-NC-ND (http:// creativecommons.org/licenses/by-nc-nd/4.0/).
\end{abstract}




\section{KEYWORDS}

Cardiac valve replacements; Biological valve

\section{Introducción}

Las enfermedades valvulares son alteraciones en la estructura valvular del corazón; normalmente, las cuatro válvulas se abren para que la sangre fluya a través o afuera del corazón en sístole y luego se cierran en diástole para impedir su retorno, pero algunas veces no funcionan de forma correcta $^{1}$. En jóvenes, la válvula más afectada es la mitral, mientras que en mayores, lo es la aórtica. La manifestación principal es la insuficiencia cardíaca, mediante síntomas y signos como disnea, fatiga, astenia y adinamia, o edema de miembros inferiores ${ }^{2}$. A su vez, la insuficiencia cardíaca varía en su espectro de presentación: leve, moderada o severa, y es en este último grupo de pacientes en quienes está indicado el tratamiento quirúrgico una vez se tornan sintomáticos ${ }^{3}$.

Las prótesis valvulares o válvulas cardíacas protésicas son dispositivos diseñados para el reemplazo de aquellas válvulas lesionadas o deterioradas, que permiten mantener el flujo unidireccional del corazón; constan de un mecanismo oclusor y de un orificio a través del cual fluye la sangre $^{4-6}$. Después de su implantación pueden desarrollarse alteraciones atribuidas a diferentes factores, como el diseño de la válvula, el material utilizado para su fabricación y el tiempo de uso; dichas alteraciones pueden clasificarse en complicaciones extrínsecas, relacionadas, por ejemplo, con la técnica quirúrgica, causadas por el tamaño inadecuado de la válvula, colocación incorrecta del poste de la estructura de las arterias coronarias, perforación inducida en la valva por una sutura excesivamente larga, fuga paravalvular y cubierta fibrosa; e, intrínsecas, que pueden tener su origen en las alteraciones periprótesis o intraprótesis, que incluyen trombosis y tromboembolia, endocarditis, hemólisis y deterioro estructural. Entre las complicaciones asociadas específicamente a las válvulas mecánicas están la tromboembolia y la necesidad de anticoagulación y de optimización de la hemodinámica. La tasa de complicaciones, como sangrado, endocarditis y trastornos de la prótesis, es muy variable y no difiere entre las distintas prótesis mecánicas ni entre las diferentes bioprótesis $^{6-8}$. Las válvulas biológicas se aconsejan en mujeres que estén en edad reproductiva ya que no requieren el uso de anticoagulación, en personas mayores de 65 años y en pacientes que tengan contraindicación para la anticoagulación; en un $70 \%$ se precisa reintervención a diez años ${ }^{9}$. En cuanto a la elección del tipo de válvula cardiaca protésica, en la actualidad se recomienda realizar un proceso de toma de decisiones compartido, que tenga en cuenta la discusión de la indicación, los riesgos de la terapia anticoagulante, el potencial riesgo de reintervención y las preferencias del paciente; en quienes son llevados a reemplazo valvular aórtico o mitral, es razonable elegir una prótesis mecánica si la edad es menor a 50 años y no existe una contraindicación para la anticoagulación; en mayores de 70 años se prefiere el uso de bioprótesis, mientras que en aquellos entre 50 y 70 años de edad se individualiza el caso y se opta por una bioprótesis o prótesis mecánica, de acuerdo con los factores individuales del paciente y sus preferencias ${ }^{10}$. 
El implante aislado de válvulas protésicas, cualquiera que sea su origen, constituye un procedimiento simple y reproducible para pacientes con enfermedad valvular grave y se asocia con una franca mejoría en la sintomatología y en la expectativa de vida ${ }^{11}$. El avance en los diferentes métodos de valoración ventricular no invasiva, el mejoramiento de las prótesis y técnicas de reconstrucción y el desarrollo de pautas para el seguimiento y la toma de decisión del momento quirúrgico ha permitido avanzar en el diagnóstico y tratamiento de los pacientes con enfermedades valvulares cardíacas $^{12}$.

\section{Justificación}

Las modificaciones en las características estructurales y funcionales de las prótesis valvulares, entre otras, han derivado en un aumento en las curvas de sobrevida/supervivencia ${ }^{13}$ de los pacientes, en una disminución en las tasas de morbilidad y mortalidad posteriores a intervenciones quirúrgicas sobre las válvulas cardíacas y en cambios sustanciales en las razones de costo-efectividad y costo-beneficio de estos procedimientos $^{14}$. La característica más importante de los datos provenientes del análisis de supervivencia es que, muy probablemente, al final del período de observación, no todos los pacientes habrán presentado el suceso objeto de estudio; además, es habitual, que se vayan incorporando nuevos pacientes durante el período de observación ${ }^{15}$. Por tanto, conocer el tiempo de supervivencia de los pacientes sometidos a reemplazo valvular cardíaco de tipo biológico puede permitir estimar el tiempo de sobrevida de futuros pacientes con enfermedad valvular sometidos a tratamiento.

\section{Pregunta guía para la revisión}

¿Cuál es la supervivencia de los pacientes de 60 años de edad sometidos a reemplazo valvular biológico durante el período 2000 a 2014 ?

\section{Objetivos}

\section{General}

- Conocer la supervivencia de pacientes de 60 y más años de edad con prótesis valvular biológica.

\section{Específicos}

- Establecer la supervivencia de pacientes con prótesis biológica aórtica.

- Identificar la supervivencia de pacientes con prótesis biológica mitral.

\section{Métodos}

Se hizo una revisión de la literatura acerca de estudios de seguimiento en los que se evaluó la supervivencia de los pacientes de 60 y más años de edad sometidos a reemplazo valvular cardíaco, mediante la búsqueda de artículos científicos registrados en la base de datos PubMed, con fundamento en los descriptores seleccionados como palabras clave. La búsqueda de los descriptores se efectuó en el programa de referencias EndNote. Se hizo la revisión de cada uno de los resúmenes y se procedió a eliminar aquellos que no correspondían con la población objeto de estudio, luego de lo cual se inició una búsqueda del texto completo del artículo a través del vínculo suministrado por el buscador y se procedió a seleccionar todos los artículos que fueran de acceso libre.

La síntesis de la evidencia - autores, título del artículo, página de web, objetivo planteado, tipo de estudio, materiales y métodos utilizados, resultados obtenidos, discusión y conclusiones - fue registrada en una hoja de cálculo Excel de Microsoft Office ${ }^{\circledR}$, diseñada para tal fin. La valoración de la fuerza de la evidencia científica de cada uno de los artículos seleccionados se llevó a cabo con base en la herramienta para evaluar la calidad de estudios cuantitativos de McMaster $^{16}$, a través de las siguientes dimensiones de clasificación: sesgo de selección, diseño del estudio, factores de confusión, cegamiento, métodos de recolección de datos, retiros y abandonos, integridad de la intervención, unidad de análisis, métodos estadísticos utilizados y análisis efectuado; cada uno clasificado como débil, moderado y fuerte según los criterios de calificación de la escala, para luego proceder a hacer la calificación global así: fuerte, cuatro dimensiones sin calificación débil; moderada, menos de cuatro dimensiones con calificación fuerte y una calificación débil; y, débil, dos o más dimensiones con calificación débil, y proceder, entonces, a seleccionar la mejor evidencia con base en los siguientes criterios.

\section{Criterio de inclusión}

Todos los estudios con calificación global fuerte y moderada, después de ser valorados con la escala de McMaster.

\section{Criterios de exclusión}

Todos los estudios con calificación global débil, según la escala de McMaster.

Para hacer la búsqueda de las referencias se utilizó el gestor de referencias EndNote ${ }^{\circledR} 7$ como medio para conectar a las bases de datos seleccionadas y hacer la importación de las referencias. Las palabras clave seleccionadas en español fueron 'Prótesis Valvulares Cardíacas', 'Bioprótesis', 'Supervivencia', y en inglés, Heart Valve Prosthesis, Survival y Bioprosthesis.

El procedimiento de búsqueda en la base de datos de PubMed mediante la utilización de operadores booleanos se ilustra en la figura 1. Una vez evaluada la fuerza de la evidencia que cumplió con los criterios de inclusión, en una base de datos diseñada en la hoja de cálculo Excel 2010 de Microsoft Office $^{\circledR}$ se registraron los datos concernientes a las variables a estudio: sexo, edad y tiempo de supervivencia, para proceder al análisis estadístico de la información recolectada mediante el uso de medidas de frecuencia, tendencia central y razón. 


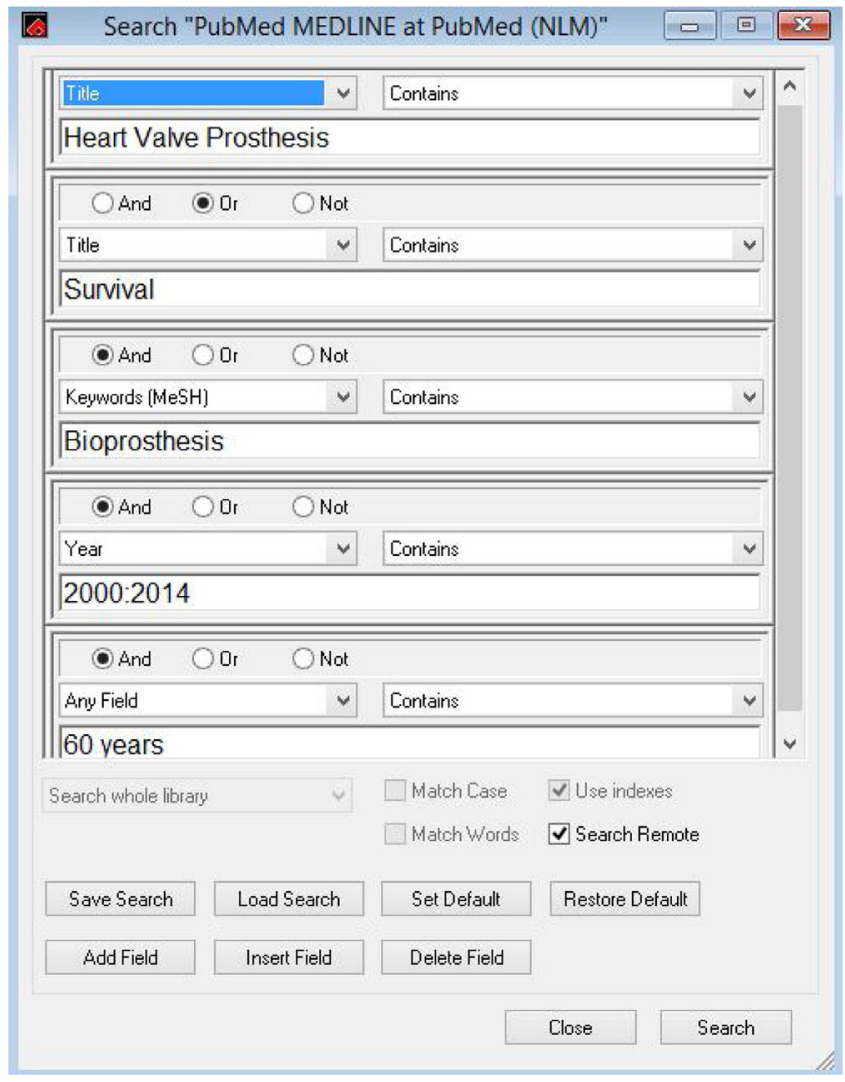

Figura 1 Procedimiento de búsqueda de referencias mediante palabras clave y operadores booleanos en la base de datos PubMed.

\section{Resultados}

De acuerdo con las palabras clave definidas en la base de datos de PubMed se obtuvo un total de 12 referencias (fig. 2), de las cuales fueron descartadas $5(41,7 \%)$ ya que no cumplían con el grupo de interés a estudio: pacientes de 60 y más años con reemplazo valvular biológico.

Después de aplicar la escala de McMaster, los siete estudios seleccionados cumplieron con los criterios de inclusión obteniendo una calificación global de "fuerte" (tabla 1). El tiempo promedio de seguimiento fue de 13,7 años con un mínimo de 6 años y un máximo de 30 años. La muestra total de pacientes intervenidos fue de 18.519; de ellos un total de $7.563(40,8 \%)$ fueron sometidos a reemplazo valvular biológico. La razón de masculinidad fue 1:0,8. La edad promedio de los pacientes fue de 67,2 años, con edad mínima de 60,0 años y máxima de 78,5 años (tabla 2).

La supervivencia de los pacientes después de ser sometidos al reemplazo valvular biológico a 5 años tuvo un promedio de $71 \%$, con una supervivencia mínima de $48 \%$ y máxima de $82 \%$, a 10 años fue de $65 \%$ con una supervivencia mínima de $38 \%$ y máxima de $85 \%$; un estudio reportó una supervivencia a un año de $88 \%$, dos estudios reportaron una supervivencia a 15 años de $51 \%$ y $61 \%$ respectivamente y un estudio reportó una supervivencia a 19 años de 45\% (tabla 2).

\section{Discusión}

De acuerdo con los operadores booleanos utilizados y al restringir la búsqueda de las palabras clave a las secciones correspondientes a título, palabras clave y período de búsqueda de publicaciones (year) entre 2000 y 2014 junto con la edad correspondiente a "60 years" en la sección "any field" únicamente se obtuvieron 12 referencias que cumplieron con estos criterios iniciales, de las cuales se descartó casi la mitad de ellas, lo que podría ser una limitación de esta revisión de la literatura. Así mismo, es importante resaltar que las siete referencias que cumplieron con los criterios de inclusión obtuvieron una calificación global fuerte después de utilizar la herramienta para evaluar la calidad de estudios cuantitativos de McMaster.

Los estudios evidencian que más de la mitad de los pacientes sometidos a reemplazo valvular biológico después de un seguimiento promedio de 13,5 años, sobreviven períodos de más de diez años. El estudio de seguimiento de Emery et al. tras treinta años arrojó una supervivencia de $82 \%$, $66 \%, 51 \%$ y $45 \%$ a los $5,10,15$ y 19 años en pacientes con prótesis biológica valvular aórtica ${ }^{17}$; en el estudio de Dellgren et al. luego de diez años de seguimiento a pacientes con estenosis aórtica sometidos a reemplazo valvular biológico se obtuvo una supervivencia actuarial tardía de $74 \%{ }^{18}$; según Roberts et al. la supervivencia de los pacientes con reemplazo valvular aórtico fue del $85 \%{ }^{19}$. Por su parte, Vicchio et al., hallaron una supervivencia a 10 años del $77 \%$ en pacientes mayores de 70 años sometidos a reemplazo valvular aórtico ${ }^{20}$; Nguyen et al., reportan una supervivencia

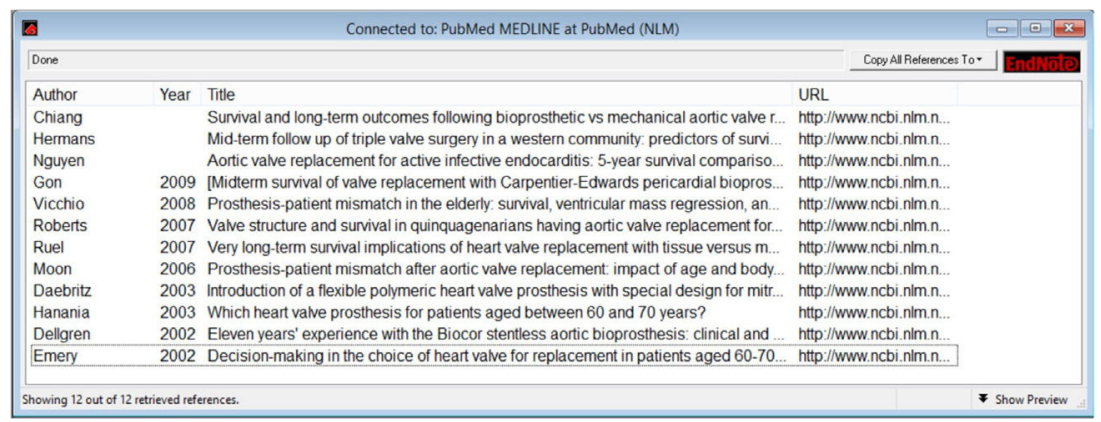

Figura 2 Listado de referencias encontradas en la base de datos PubMed. 
Tabla 1 Resultados de calificación de la evidencia con la escala de McMaster

\begin{tabular}{|c|c|c|c|c|c|c|c|c|}
\hline Título completo & Autores & $\begin{array}{l}\text { Sesgo de } \\
\text { selección de } \\
\text { la muestra }\end{array}$ & $\begin{array}{l}\text { Diseño de } \\
\text { estudio/ } \\
\text { Tipo estudio }\end{array}$ & Confusores & Cegamiento & $\begin{array}{l}\text { Métodos de } \\
\text { recopilación } \\
\text { de datos }\end{array}$ & $\begin{array}{l}\text { Retiros y } \\
\text { abandonos/ } \\
\text { participación }\end{array}$ & $\begin{array}{l}\text { Calificación } \\
\text { global de la } \\
\text { evidencia }\end{array}$ \\
\hline $\begin{array}{l}\text { Decision-making in the choice of } \\
\text { heart valve for replacement in } \\
\text { patients aged } 60-70 \text { years: } \\
\text { twenty-year follow up of the St. } \\
\text { Jude Medical aortic valve } \\
\text { prosthesis }\end{array}$ & $\begin{array}{l}\text { Emery RW, Arom KV, } \\
\text { Kshettry VR, Kroshus TJ, } \\
\text { Von R, Kersten TE, et al }\end{array}$ & 1 & 1 & No aplica & No aplica & 1 & 1 & Fuerte \\
\hline $\begin{array}{l}\text { Eleven years' experience with the } \\
\text { Biocor stentless aortic } \\
\text { bioprosthesis: clinical and } \\
\text { hemodynamic follow-up with } \\
\text { long-term relative survival rate }\end{array}$ & $\begin{array}{l}\text { Dellgren G, Eriksson MJ, } \\
\text { Brodin LA, Rådegran K }\end{array}$ & 1 & 1 & No aplica & No aplica & 1 & 1 & Fuerte \\
\hline $\begin{array}{l}\text { Valve structure and survival in } \\
\text { quinquagenarians having aortic } \\
\text { valve replacement for aortic } \\
\text { stenosis (+/- aortic } \\
\text { regurgitation) with versus } \\
\text { without coronary artery bypass } \\
\text { grafting at a single US medical } \\
\text { center (1993 to 2005) }\end{array}$ & $\begin{array}{l}\text { Roberts WC, Ko JM, Filardo } \\
\text { G, Henry AC, Hebeler RF, } \\
\text { Jr., Cheung EH-K, et al }\end{array}$ & 1 & 1 & No aplica & No aplica & 1 & 1 & Fuerte \\
\hline $\begin{array}{l}\text { Scardone } M \text {, et al. } \\
\text { Prosthesis-patient mismatch in } \\
\text { the elderly: survival, ventricular } \\
\text { mass regression, and quality of } \\
\text { life }\end{array}$ & $\begin{array}{l}\text { Vicchio M, Della Corte A, De } \\
\text { Santo LS, De Feo M, } \\
\text { Caianiello G, Scardone M, } \\
\text { et al }\end{array}$ & 1 & 1 & No aplica & No aplica & 1 & 1 & Fuerte \\
\hline $\begin{array}{l}\text { Aortic valve replacement for } \\
\text { active infective endocarditis: } \\
5 \text {-year survival comparison of } \\
\text { bioprostheses, homografts and } \\
\text { mechanical prostheses }\end{array}$ & $\begin{array}{l}\text { Nguyen DT, Delahaye F, } \\
\text { Obadia J-F, Duval X, } \\
\text { Selton-Suty C, Carteaux J-P, } \\
\text { et al }\end{array}$ & 1 & 1 & No aplica & No aplica & 1 & 1 & Fuerte \\
\hline $\begin{array}{l}\text { Mid-term follow up of triple valve } \\
\text { surgery in a western } \\
\text { community: predictors of } \\
\text { survival }\end{array}$ & $\begin{array}{l}\text { Hermans } \mathrm{H} \text {, Tjahjono } \mathrm{M} \text {, } \\
\text { Faes D, Belmans A, Meuris } \\
\mathrm{B} \text {, Herijgers } \mathrm{P} \text {, et al }\end{array}$ & 1 & 1 & No aplica & No aplica & 1 & 1 & Fuerte \\
\hline $\begin{array}{l}\text { Survival and long-term outcomes } \\
\text { following bioprosthetic vs } \\
\text { mechanical aortic valve } \\
\text { replacement in patients aged } 50 \\
\text { to } 69 \text { years }\end{array}$ & $\begin{array}{l}\text { Chiang YP, Chikwe J, } \\
\text { Moskowitz AJ, Itagaki S, } \\
\text { Adams DH, Egorova NN }\end{array}$ & 1 & 1 & No aplica & No aplica & 1 & 1 & Fuerte \\
\hline
\end{tabular}


Tabla 2 Distribución de resultados de los estudios seleccionados según las variables definidas

\begin{tabular}{|c|c|c|c|c|c|c|c|c|c|c|c|c|c|}
\hline \multirow[t]{2}{*}{ Título completo } & \multirow[t]{2}{*}{ Autores } & \multirow{2}{*}{$\begin{array}{l}\text { Tiempo de } \\
\text { seguimiento } \\
\text { en años }\end{array}$} & \multirow{2}{*}{$\begin{array}{l}\text { Total } \\
\text { pacientes } \\
\text { intervenidos }\end{array}$} & \multirow{2}{*}{$\begin{array}{l}\text { Total pacientes } \\
\text { con reemplazo } \\
\text { valvular } \\
\text { biológico }\end{array}$} & \multirow[t]{2}{*}{ Hombres } & \multirow[t]{2}{*}{ Mujeres } & \multirow[t]{2}{*}{$\begin{array}{l}\text { Edad } \\
\text { media }\end{array}$} & \multirow[t]{2}{*}{$\begin{array}{l}\text { Desviación } \\
\text { típica }\end{array}$} & \multirow[t]{2}{*}{ Rango } & \multicolumn{4}{|c|}{$\begin{array}{l}\text { \% de Supervivencia } \\
\text { en años }\end{array}$} \\
\hline & & & & & & & & & & 5 & 10 & 15 & 19 \\
\hline $\begin{array}{l}\text { Decision-making in } \\
\text { the choice of } \\
\text { heart valve for } \\
\text { replacement in } \\
\text { patients aged } \\
60-70 \text { years: } \\
\text { twenty-year } \\
\text { follow up of the } \\
\text { St. Jude Medical } \\
\text { aortic valve } \\
\text { prosthesis }\end{array}$ & $\begin{array}{l}\text { Emery RW, } \\
\text { Arom KV, } \\
\text { Kshettry VR, } \\
\text { Kroshus TJ, Von } \\
\text { R, Kersten TE, } \\
\text { et al }\end{array}$ & 20 & 13.208 & 2.390 & 1.500 & 890 & 63 & \pm 14 & & 82 & 66 & 51 & 45 \\
\hline $\begin{array}{l}\text { Eleven years' } \\
\text { experience with } \\
\text { the Biocor } \\
\text { stentless aortic } \\
\text { bioprosthesis: } \\
\text { clinical and } \\
\text { hemodynamic } \\
\text { follow-up with } \\
\text { long-term } \\
\text { relative survival } \\
\text { rate }\end{array}$ & $\begin{array}{l}\text { Dellgren G, } \\
\text { Eriksson MJ, } \\
\text { Brodin LA, } \\
\text { Rådegran } \mathrm{K}\end{array}$ & 10 & 112 & 112 & 38 & 74 & 78,5 & & $60-80$ & 74 & 38 & & \\
\hline
\end{tabular}




\begin{tabular}{|c|c|c|c|c|c|c|c|c|c|c|c|c|c|}
\hline Título completo & Autores & $\begin{array}{l}\text { Tiempo de } \\
\text { seguimiento } \\
\text { en años }\end{array}$ & $\begin{array}{l}\text { Total } \\
\text { pacientes } \\
\text { intervenidos }\end{array}$ & $\begin{array}{l}\text { Total pacientes } \\
\text { con reemplazo } \\
\text { valvular } \\
\text { biológico }\end{array}$ & Hombres & Mujeres & $\begin{array}{l}\text { Edad } \\
\text { media }\end{array}$ & $\begin{array}{l}\text { Desviación } \\
\text { típica }\end{array}$ & Rango & \multicolumn{4}{|c|}{$\begin{array}{l}\text { \% de Supervivencia } \\
\text { en años }\end{array}$} \\
\hline $\begin{array}{l}\text { Valve structure } \\
\text { and survival in } \\
\text { quinquagena- } \\
\text { rians having } \\
\text { aortic valve } \\
\text { replacement for } \\
\text { aortic stenosis } \\
\text { (+/-aortic } \\
\text { regurgitation) } \\
\text { with versus } \\
\text { without } \\
\text { coronary artery } \\
\text { bypass grafting } \\
\text { at a single US } \\
\text { medical center } \\
\text { (1993 to 2005) }\end{array}$ & $\begin{array}{l}\text { Roberts WC, Ko } \\
\text { JM, Filardo G, } \\
\text { Henry AC, } \\
\text { Hebeler RF, Jr., } \\
\text { Cheung EH-K, } \\
\text { et al }\end{array}$ & 12 & 120 & 120 & & & $>50$ & $>50$ & & & 85 & & \\
\hline
\end{tabular}




\begin{tabular}{|c|c|c|c|c|c|c|c|c|c|c|c|c|c|}
\hline \multirow[t]{2}{*}{ Título completo } & \multirow[t]{2}{*}{ Autores } & \multirow{2}{*}{$\begin{array}{l}\text { Tiempo de } \\
\text { seguimiento } \\
\text { en años }\end{array}$} & \multirow{2}{*}{$\begin{array}{l}\text { Total } \\
\text { pacientes } \\
\text { intervenidos }\end{array}$} & \multirow{2}{*}{$\begin{array}{l}\text { Total pacientes } \\
\text { con reemplazo } \\
\text { valvular } \\
\text { biológico }\end{array}$} & \multirow[t]{2}{*}{ Hombres } & \multirow[t]{2}{*}{ Mujeres } & \multirow[t]{2}{*}{$\begin{array}{l}\text { Edad } \\
\text { media }\end{array}$} & \multirow[t]{2}{*}{$\begin{array}{l}\text { Desviación } \\
\text { típica }\end{array}$} & \multirow[t]{2}{*}{ Rango } & \multicolumn{4}{|c|}{$\begin{array}{l}\text { \% de Supervivencia } \\
\text { en años }\end{array}$} \\
\hline & & & & & & & & & & 5 & 10 & 15 & 19 \\
\hline $\begin{array}{l}\text { Aortic valve } \\
\text { replacement for } \\
\text { active infective } \\
\text { endocarditis: } \\
\text { 5-year survival } \\
\text { comparison of } \\
\text { bioprostheses, } \\
\text { homografts and } \\
\text { mechanical } \\
\text { prostheses }\end{array}$ & $\begin{array}{l}\text { Nguyen DT, } \\
\text { Delahaye F, } \\
\text { Obadia J-F, } \\
\text { Duval X, } \\
\text { Selton-Suty C, } \\
\text { Carteaux J-P, } \\
\text { et al }\end{array}$ & 7 & 283 & 177 & & & 63,2 & & & 48 & & & \\
\hline $\begin{array}{l}\text { Mid-term follow } \\
\text { up of triple } \\
\text { valve surgery in } \\
\text { a western } \\
\text { community: } \\
\text { predictors of } \\
\text { survival }\end{array}$ & $\begin{array}{l}\text { Hermans } \mathrm{H}, \\
\text { Tjahjono M, } \\
\text { Faes D, } \\
\text { Belmans A, } \\
\text { Meuris B, } \\
\text { Herijgers P, } \\
\text { et al }\end{array}$ & 6 & 166 & 166 & 34 & 132 & 64 & & $55-71$ & 70 & 60 & & \\
\hline $\begin{array}{l}\text { Survival and } \\
\text { long-term } \\
\text { outcomes } \\
\text { following } \\
\text { bioprosthetic vs } \\
\text { mechanical } \\
\text { aortic valve } \\
\text { replacement in } \\
\text { patients aged } 50 \\
\text { to } 69 \text { years }\end{array}$ & $\begin{array}{l}\text { Chiang YP, } \\
\text { Chikwe J, } \\
\text { Moskowitz AJ, } \\
\text { Itagaki S, } \\
\text { Adams DH, } \\
\text { Egorova NN }\end{array}$ & 11 & 4.253 & 4.253 & & & 60 & & & & & 61 & \\
\hline
\end{tabular}


de $48 \%$ a cinco años en pacientes con edad promedio de 63 años con reemplazo valvular aórtico ${ }^{21}$; Hermans et al., encontraron una supervivencia a 10 años del $60 \%$ en pacientes sometidos a cirugía valvular triple de tipo biológico con edad promedio de 64 años $^{22}$; y el estudio de Chiang et al., encontró una supervivencia a 15 años de $61 \%$ en pacientes sometidos a reemplazo valvular aórtico ${ }^{23}$.

En los estudios no hay diferencias en cuanto a la supervivencia relacionada con el sexo de los pacientes, pero en algunos se evidencia mayor supervivencia entre los pacientes sometidos a reemplazo valvular mecánico versus el biológico, y plantean, entonces, como una opción razonable, el uso de prótesis biológicas en pacientes mayores de 60 años; así mismo, se reconoce la necesidad de hacer más estudios que puedan corroborar sus hallazgos.

Cabe resaltar que esta revisión fue hecha como un ejercicio académico con las limitaciones que puede esto representar, entre otras, la de haber delimitado la búsqueda de las palabras clave al título y el período de tiempo de consulta. Por tanto, se recomienda ampliar la búsqueda de las palabras clave, por ejemplo, en la sección del resumen o en todo el texto del manuscrito, lo cual podría permitir contar con un mayor número de artículos que permitan corroborar los hallazgos hechos. Podría también incluirse en la búsqueda la marca registrada de las válvulas biológicas y así contar con una comparación de cuál de ellas podría ser más efectiva o incluir los datos ecocardiográficos de seguimiento de los pacientes.

\section{Responsabilidades éticas}

Protección de personas y animales. Los autores declaran que para esta investigación no se han realizado experimentos en seres humanos ni en animales.

Confidencialidad de los datos. Los autores declaran que en este artículo no aparecen datos de pacientes.

Derecho a la privacidad y consentimiento informado. Los autores declaran que en este artículo no aparecen datos de pacientes.

\section{Financiación}

Recursos propios.

\section{Conflictos de interés}

Ninguno.

\section{Bibliografía}

1. NIH: Instituto Nacional del Corazón IPyIS. Enfermedades de las válvulas del corazón 2014 [Accerso 16 Oct 2014]. Disponible en: https: / /medlineplus.gov/spanish/heartvalvediseases.html.

2. Rábago G. Enfermedades valvulares: Clínica Universidad de Navarra. 2013 [Acceso 16 Oct 2014]. Disponible en: https://www.cun.es/enfermedades-tratamientos/ enfermedades/enfermedades-valvulares.

3. Nishimura RA, Otto C, Bonow R, Carabello B, Guyton RA, O'Gara PT, et al. 2014 AHA/ACC guideline for the management of patients with valvular heart disease: a report of the American College of Cardiology/American Heart Association task force on practice guidelines. Circulation. 2014;129:e521-643.

4. Álvarez HR. Válvulas cardiacas protésicas. Revisión actualizada. Revista de Postgrado de la VIa Cátedra de Medicina. 2004;137:19-32.

5. Azpitarte J, Alonso AM, Gallego FG, Santos JMG, Paré C, Tello A. Guías de práctica clínica de la Sociedad Española de Cardiología en Valvulopatías. Rev Esp Cardiol. 2000;53:1209-78.

6. Villar Inclán A. Válvulas cardíacas protésicas: revisión histórica del tema. Rev Cubana Cir. 2010:49.

7. Ferrans VJ, Loredo ML. Complicaciones de las válvulas protésicas cardiacas. Archivos de Cardiología de México. 2001;71: $10-2$.

8. Garfia A, Martínez M, Repetto F. Evaluación médico-legal de las prótesis intracardíacas en la autopsia: II. Prótesis valvulares. Cuadernos de Medicina Forense. 2002:61-6.

9. Guerra P. Tratamiento quirúrgico de la valvulopatía mitral. En: Charria DJ, Guerra P, Manzur F, Llamas A, Rodríguez NI, Sandoval NF, et al., editores. Texto de Cardiología. Bogotá, D.C: Sociedad Colombiana de Cardiología y Cirugía Cardiovascular; 2007. p. 569-78.

10. Mendoza F, Pérez OA. Actualización guías AHA/ACC enfocadas en el manejo de los pacientes con Enfermedad Valvular Cardíaca: 10 puntos actuales para recordar en el manejo óptimo del paciente con Valvulopatía: Boletín Puesta al día en Cardiología. Sociedad Colombiana de Cardiología;. 2015 [Acceso 17 Mar 2019]. Disponible en: http://scc.org.co/wpcontent/uploads/2015/11/BOLETIN-No-102.pdf.

11. López LJ. Válvulas cardíacas: Funcionamiento y enfermedades. En: López A, Macaya C, editores. Libro de la salud cardiovascular. Bilbao: Hospital Clínico San Carlos y la Fundación BBVA; 2009. p. 467-9.

12. Salazar G, Rincón OS, Cely A. Enfermedades de la válvula aórtica. En: Charria DJ, Guerra P, Manzur F, Llamas A, Sandoval NF, Melgarejo E, editores. Texto de Cardiología. Bogotá, D.C: Sociedad Colombiana de Cardiología y Cirugía Cardiovascular; 2007. p. 531-44.

13. Rebasa P. Conceptos básicos del análisis de supervivencia. Cirugía Española. 2005;78:222-30.

14. Parra TE. Prótesis valvulares cardíacas. En: Charria DJ, Guerra PG, Manzur F, Llamas A, Rodríguez NI, Sandoval NF, et al, editores. Texto de Cardiología. Bogotá, D.C: Sociedad Colombiana de Cardiología y Cirugía Cardiovascular; 2007. p. 599-607.

15. Arribalzaga EB. Interpretación de las curvas de supervivencia. Revista Chilena de Cirugía. 2007;59:75-83.

16. McMaster University, School of Nursing. Quality assessment tool for quantitative studies 2010 [Acceso 28 Agt 2014]. Disponible en: https://dev.nccmt.ca/registry/resource/pdf/14.pdf.

17. Emery RW, Arom KV, Kshettry VR, Kroshus TJ, Von R, Kersten TE, et al. Decision-making in the choice of heart valve for replacement in patients aged 60-70 years: twenty-year follow up of the St. Jude Medical aortic valve prosthesis. The Journal of Heart Valve Disease. 2002;11 Suppl 1:S37-44.

18. Dellgren G, Eriksson MJ, Brodin LA, Rådegran K. Eleven years' experience with the Biocor stentless aortic bioprosthesis: clinical and hemodynamic follow-up with long-term relative survival rate. 2002;22:912-21.

19. Roberts WC, Ko JM, Filardo G, Henry AC, Hebeler RF Jr, Cheung $\mathrm{EH}-\mathrm{K}$, et al. Valve structure and survival in quinquagenarians having aortic valve replacement for aortic stenosis (+/-aortic regurgitation) with versus without coronary artery bypass grafting at a single US medical center (1993 to 2005). Am J Cardiol. 2007;100:1584-91.

20. Vicchio M, Della Corte A, De Santo LS, De Feo M, Caianiello G, Scardone $M$, et al. Prosthesis-patient mismatch in the elderly: survival, ventricular mass regression, and quality of life. Ann Thorac Surg. 2008;86:1791-7. 
21. Nguyen DT, Delahaye F, Obadia J-F, Duval X, Selton-Suty C, Carteaux J-P, et al. Aortic valve replacement for active infective endocarditis: 5-year survival comparison of bioprostheses, homografts and mechanical prostheses. Eur J Cardiothorac Surg. 2010;37:1025-32.

22. Hermans H, Tjahjono M, Faes D, Belmans A, Meuris B, Herijgers $\mathrm{P}$, et al. Mid-term follow up of triple valve surgery in a western community: predictors of survival. J Heart Valve Dis. 2010;19:644-51.

23. Chiang YP, Chikwe J, Moskowitz AJ, Itagaki S, Adams DH, Egorova NN. Survival and long-term outcomes following bioprosthetic vs mechanical aortic valve replacement in patients aged 50 to 69 years. JAMA. 2014;312:1323-9. 